\title{
DPP4 Polymorphisms Are Associated With Hypoalphalipoproteine mia and DPP4 Serum Levels. A Case-control Study of the GEA Cohort.
}

\section{Gilberto Vargas-Alarcón}

Departamento de Biología Molecular, Instituto Nacional de Cardiología Ignacio Chávez, Ciudad de Mexico 14080, Mexico

\section{María del Carmen González-Salazar}

Departamento de Endocrinologia, Instituto Nacional de Cardiología Ignacio Chávez, Ciudad de Mexico 14080, Mexico

\section{Christian Vázquez-Vázquez}

Departamento de Biología Molecular, Instituto Nacional de Cardiología Ignacio Chávez, Ciudad de Mexico 14080, Mexico

\section{Adrián Hernández-Díaz Couder}

Departamento de Inmunología, Instituto Nacional de Cardiología Ignacio Chávez, Ciudad de Mexico 14080, Mexico

\section{Fausto Sánchez-Muñoz}

Departamento de Inmunología, Instituto Nacional de Cardiología Ignacio Chávez, Ciudad de Mexico 14080, Mexico

\section{Juan Reyes-Barrera}

Departamento de Endocrinologia, Instituto Nacional de Cardiología Ignacio Chávez, Ciudad de Mexico 14080, Mexico

\section{Sergio A. Criales-Vera}

Departamento de Tomografía, Instituto Nacional de Cardiología Ignacio Chávez, Ciudad de Mexico 14080, Mexico

\section{Marco Sánchez-Guerra}

Departamento de Neurobiologia del Desarrollo, Instituto Nacional de Perinatologia, Ciudad de Mexico 11000, Mexico

\section{Citlalli Osorio-Yáñez}

Instituto de Investigaciones Biomédicas, Universidad Nacional Autónoma de Mexico, Ciudad de Mexico 70228, Mexico

\section{Rosalinda Posadas-Sanchez ( $\sim$ rossy_posadas_s@yahoo.it )}

Instituto Nacional de Cardiologia Ignacio Chavez

\section{Research}


Keywords: Dipeptidyl peptidase-4, hypoalphalipoproteinemia, insulin resistance, hyperinsulinemia, polymorphism, DPP4 serum levels

Posted Date: June 25th, 2020

DOI: https://doi.org/10.21203/rs.3.rs-37788/v1

License: (c) (1) This work is licensed under a Creative Commons Attribution 4.0 International License. Read Full License 


\section{Abstract}

Background: Dipeptidyl peptidase-4 (DPP4) can influence lipid homeostasis and atherosclerosis progression. Our aim was to assess the association of DPP4 gene polymorphisms with hypoalphalipoproteinemia and DPP4 levels, in a cohort of Mexican individuals.

Methods: Five DPP4 polymorphisms (rs12617336, rs12617656, rs1558957, rs3788979, rs17574) were genotyped in 748 participants with and 745 without hypoalphalipoproteinemia. The associations were evaluated using logistic regression analyses.

Results: Under inheritance models adjusted for confounding variables, the rs12617336 (OR $=0.22$, $\left.P_{\text {heterozygote }}=0.001\right)$ and $r s 17574\left(\mathrm{OR}=0.79, \mathrm{P}_{\text {additive }}=0.033 ; \mathrm{OR}=0.75, \mathrm{P}_{\text {dominant }}=0.021 ; \mathrm{OR}=0.75\right.$, $\left.P_{\text {heterozygote }}=0.029 ; O R=0.74, P_{\text {codominant } 1}=0.024\right)$ polymorphisms were associated with a low risk of hypoalphalipoproteinemia. Moreover, GTTCG haplotype was also related with a low risk of this condition $(\mathrm{OR}=0.75, \mathrm{P}=0.021)$. Additionally, both polymorphisms were associated with protection for the presence of insulin resistance $(I R)\left(O R=0.17, P_{\text {heterozygote }}=0.019\right.$ for $r s 12617336$ and $O R=0.75, P_{\text {additive }}=0.049$ for rs17574). The rs12617336 was also associated with a low risk of hyperinsulinemia $(O R=0.11$, $P_{\text {heterozygote }}=0.006$ ). Difference in DPP4 levels were observed in individuals with rs 17574 genotypes, the rs 17574 GG genotype individuals had the lowest levels.

Conclusions: Our data suggest that rs12617336 and rs17574 DPP4 polymorphism could be envisaged as protective genetic markers for hypoalphalipoproteinemia, IR, and hyperinsulinemia. The rs $17574 G G$ genotype was associated with the lowest DPP4 levels.

\section{Background}

Hyperglycemia, insulin resistance (IR), dyslipidemia, oxidative stress, and inflammation are welldocumented risk factors for subclinical atherosclerosis (SA) and cardiovascular disease (CVD). Atherogenic dyslipidemia is characterized by an increase in triglyceride-rich lipoproteins, low high-density lipoprotein (HDL) cholesterol (HDL-C) levels, accumulation of lipoprotein remnants, and dense lowdensity lipoprotein (LDL) particles[1, 2]. According to results of the National Health and Nutrition Survey 2012, hypoalphalipoproteinemia (HA) and elevated LDL-cholesterol (LDL-C) are the most prevalent dyslipidemias among Mexican adults [3]. Decreased levels of serum HDL-C $(<40 \mathrm{mg} / \mathrm{dl}$ in men and < $50 \mathrm{mg} / \mathrm{dl}$ in women) have been found to be a very significant independent risk factor for atherosclerotic CVD $[4,5]$. Some genetic variants in different genes such as lipoprotein lipase, cholesteryl ester transfer protein, and ATP binding cassette transporter A-I have been associated with low HDL-C levels in patients with CVD[6-8].

Dipeptidyl peptidase-4 (DPP4, also known as CD26) is a multifunctional protein expressed in two molecular forms: a soluble form circulating in the plasma, and a membrane-anchored form. Both forms have peptidase activity and cleave a wide variety of substrates, such as incretins (GLP-1, glucagon-like 
peptide-1, and GIP, glucose-dependent insulinotropic polypeptide), chemokines, growth factors and regulatory peptides[9]. Recent studies have shown the participation of DPP4 in the pathogenesis of hyperglycemia, IR, dyslipidemia, oxidative stress, inflammation[10-13], fatty liver (FL)[14] and SA[15]. Moreover, DPP4 is an adipokine with increased expression in obesity[10, 16], FL[17], and type 2 diabetes mellitus (T2DM)[18] abnormalities that coexist with chronic inflammation. Several single nucleotide polymorphisms (SNPs) have been described in the DPP4 gene (located in region 2q24.3), some of which have been associated with rheumatoid arthritis[19], T2DM, variation in DPP4[20] and apolipoprotein B (apo B) levels[21], and with increased risk of myocardial infarction in patients with coronary artery disease (CAD)[22]. Considering the high prevalence of obesity and comorbidities such as T2DM[23] and HA in the Mexican population[3] and that the expression of DPP4 is increased in these abnormalities, the aim of the present study was to evaluate the association of the DPP4 polymorphisms with the presence of HA and with DPP4 levels in a cohort of Mexican individuals. Based on the results obtained from a functional prediction analysis, we decide to study 5 DPP4 polymorphisms (rs12617336, rs 12617656, rs1558957, rs3788979, and rs17574) with possible functional consequences and/or with minor allele frequency $>5 \%$.

\section{Methods}

\section{Study population}

The study was conducted in accordance with the Declaration of Helsinki and was approved by the Ethics Committee of the Instituto Nacional de Cardiología Ignacio Chávez (Project number 18-1082). All participants gave their written informed consent for inclusion before they participated in the study. All the participants were selected from the GEA Mexican study. This project was designed to examine the premature $C A D(p C A D)$ genetic bases and the association with emerging and traditional cardiovascular risk factors in the Mexican population. All study subjects were not blood-related and were Mexican mestizos defined as a person who was born in Mexico and whose last two ascending generations were also born in Mexico. This report is a cross-sectional analysis of the baseline evaluation of the GEA study control group; this group includes 1493 individuals without either PCAD personal or family history, enrolled from social service centers, and blood banks. In the whole sample, demographic, family and personal medical history, nutritional and smoking habits, physical activity, clinical, anthropometric and biochemical variables and cardiovascular risk factors were evaluated and defined as previously described[24-26]. Briefly, BMI was calculated as weight in kilograms divided by height in square meters, and obesity was defined when $\mathrm{BMI} \geq 30 \mathrm{~kg} / \mathrm{m} 2$. Waist circumference was measured using a glass fiber measuring tape in the middle point of the distance between the lower side of the waist and the iliac crest. Criteria from the American Heart Association National Heart, Lung, and Blood Institute Scientific Statement on the Metabolic Syndrome were used in order to define HA and hypertriglyceridemia[27]. High non-HDL-C was defined when its values were $>160 \mathrm{mg} / \mathrm{dL}$. High apoB levels were considered when its values were $>110 \mathrm{mg} / \mathrm{dL}[28]$. LDL and HDL particle size was estimated with the LDL-C/apoB and HDL$\mathrm{C} /$ apoA ratios, respectively. HDL estimated diameter was calculated as described by Mazer et al[29]. LDL 
pattern B was considered with an LDL-C/apoB ratio of 1.2, which corresponded to an LDL diameter of $25.5 \mathrm{~nm}$, which is the cut-off value to distinguishing LDL pattern $A$ from pattern $B[30]$. When individuals self-reported current use of cigarettes, they were considered as current smoking. Type 2 diabetes mellitus was defined by the American Diabetes Association criteria, with a fasting glucose $\geq 126 \mathrm{mg} / \mathrm{dL}$ and was also considered when participants reported glucose-lowering treatment or a physician diagnosis of diabetes. Elevated hsCRP was considered when its values were $\geq 3 \mathrm{mg} / \mathrm{L}$. Hyperuricemia was considered with a serum uric acid $>6.0 \mathrm{mg} / \mathrm{dl}$ and $>7.0 \mathrm{mg} / \mathrm{dl}$ for women and men[31], respectively. HI was defined when insulin concentration was $\geq 75$ th percentile $(16.97 \mu \mathrm{U} / \mathrm{mL}$ in women and $15.20 \mu \mathrm{U} / \mathrm{mL}$ in men). IR was estimated using the HOMA-IR. The presence of IR was considered when the HOMA-IR values were $\geq$ 75th percentile (3.66 in women and 3.38 in men). These cutoff points were obtained from a GEA Mexican study sample of 131 men and 185 women without obesity and with normal values of blood pressure, fasting glucose, and lipids. VAF area was quantified as described by Kvist et al.,[32] and the CAC score using the Agatston method[33] after performed computed tomography of the chest and abdomen. Physical activity was measured using the Baecke questionnaire[34]. Total activity was obtained from the sum of the work exercise and leisure time activities. This questionnaire has been validated in adult populations and provides reliable information.

\section{Quantification Of Dpp4 Concentration}

In all participants, DPP4 serum concentration was quantified using a Bioplex system (R\&D Systems, Minneapolis, USA) according to the manufacturer's instructions. The data were analyzed using the BioPlex Manager software. Results are expressed in $\mathrm{ng} / \mathrm{mL}$.

\section{Genotyping}

We used standard techniques for the isolation of genomic DNA from whole blood containing EDTA. The possible functional effect of the DPP4 SNPs was defined using bioinformatics tools. For the analysis, we selected five DPP4 gene SNPs with minor allele frequencies $>5 \%$ and/or possible functional consequences. Using 5 ' exonuclease TaqMan genotyping assays, the rs 12617336, rs 12617656 , rs1558957, rs3788979, and rs17574 DPP4 SNPs were genotyped on an ABI Prism 7900HT Fast RealTime PCR system (Applied Biosystems, Foster City, CA).

\section{Statistical analysis}

Data are expressed as frequencies, median (interquartile range) or mean \pm standard deviation, as appropriate. Either Mann-Whitney U or Student's t-test was used for continuous variable comparisons, while the chi-squared test was employed for categorical variable comparisons. The frequencies of alleles and genotypes were determined by direct counting. We used the chi-squared test to determine the HardyWeinberg's equilibrium. Haplotype analysis and linkage disequilibrium were performed with Haploview software (version 4.1, Broad Institute of Massachusetts Institute of Technology and Harvard University, 
Cambridge, MA, USA). DPP4 serum concentration comparisons were evaluated by the Mann-Whitney U test or Kruskal Wallis test, as appropriate. We used logistic regression analysis (adjusted for confounding variables as appropriate) to test for the relation of studied SNPs with HA, SA, and cardiovascular risk factors. The analysis of association with HA and each cardiovascular risk factor, for each polymorphism and each inheritance model, was done individually. Therefore, no adjustment is required for multiple comparisons. A value of $\mathrm{P}<0.05$ was considered significant. All analyses were performed using SPSS software v15.0 (SPSS Chicago, IL).

\section{Results}

\section{Study sample characteristics}

One thousand four hundred ninety-three individuals belonging to the Genetics of the Atherosclerosis Disease (GEA) Mexican study control group, were included in the present analysis. According to the HDLC levels, 748 participants were $\mathrm{HA}(<40 \mathrm{mg} / \mathrm{dl}$ in men and $<50 \mathrm{mg} / \mathrm{dl}$ in women), while 745 individuals were considered non-HA. Table 1 shows demographic, lifestyle, clinical, and biochemical characteristics as well as the tomographic data and study population genotypes. Age, percentage of male, waist circumference, and the presence of T2DM were similar in both groups. Compared with non-HA individuals, body mass index (BMI), triglycerides and apoB levels, visceral adipose fat (VAF), and the prevalence of hyperinsulinemia $(\mathrm{HI})$, IR, hyperuricemia, high $\mathrm{C}$ reactive protein (hsCRP), high non-highdensity lipoprotein cholesterol (non-HDL-C), high apoB levels, LDL pattern $B$, and current smoking were significantly higher in HA subjects. On the contrary, HDL-C, apolipoprotein A (apoA) and DPP4 concentration, HDL diameter, HDL and LDL size, physical activity, and the percentage of rs $12617336 \mathrm{GC}$ and rs17574GG genotypes were higher in non-HA subjects than in HA ones (Table 1). 
Table 1

Demographic, life style, clinical characteristics, lipid profile, tomographic data, and genotypes in the studied groups.

\section{Hypoalphalipoproteinemia}

$$
\text { No }(n=745) \quad \text { Yes }(n=748) \quad \text { *P value }
$$

\section{Demographic}

Age (years)
Sex (\% male)
Clinical characteristics and coronary risk
factors

Body mass index $(\mathrm{kg} / \mathrm{m} 2)$

Waist circumference $(\mathrm{cm})$

Type 2 diabetes mellitus (\%)

Hyperinsulinemia (\%)

Insulin resistance (\%)

Hyperuricemia (\%)

High sensitivity $C$ reactive protein $\geq 3 \mathrm{mg} / \mathrm{L}$

Non-HDL-cholesterol > $160 \mathrm{mg} / \mathrm{dL}$

High apolipoprotein B

\section{Lipid profile and DPP4 concentration}

HDL-cholesterol

Triglycerides $(\mathrm{mg} / \mathrm{dL})$

Apolipoprotein A

Apolipoprotein B

Estimated HDL size

Estimated HDL diameter

Estimated LDL size

Pattern B (\%)
$54 \pm 9$

50.7
$52 \pm 9$

49.7
0.088

0.368
$27.1[24.6-29.9] \quad 28.6[26.4-31.4]<0.001$

$96.6 \pm 11.3 \quad 96.5 \pm 10.7 \quad 0.879$

46.2

53.8

0.283

45.7

63.5

$<0.001$

47.5

66.7

$<0.001$

19.1

25.5

0.003

23.3

30.8

0.001

28.9

34.3

0.026

25.9

34.3

$<0.001$

Data are shown as mean \pm standard deviation, median [interquartile range] or percentage. *Student's $t$ test, Mann Whitney's U test or Chi square test. HDL: High density lipoprotein, LDL: low density lipoprotein. 


\section{Hypoalphalipoproteinemia}

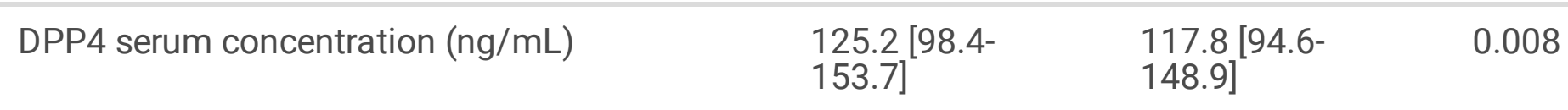

\section{Tomography}

Visceral abdominal fat $(\mathrm{cm} 2)$

$134[98-186]$

159 [124-201]

$<0.001$

\section{Life style}

Current smoking habit (\%)

19.5

25.2

0.009

Physical activity

$8.0[7.1-8.9]$

$7.8[6.9-8.6]$

0.002

DPP4 genotype (\%)

rs12617336 (GG/GC/CC)

$96.5 / 3.5 / 0$

$98.8 / 1.2 / 0$

0.003

rs12617656 (CC/CT/TT)

38.8/47.4/13.8

$36.7 / 46.5 / 54.8$

0.276

rs1558957 (CC/CT/TT)

29.0/51.5/19.5

$31.3 / 49.5 / 19.2$

0.603

rs3788979 (CC/CT/TT)

$50.5 / 40.1 / 9.5$

$46.5 / 43.8 / 9.8$

0.286

rs17574 (AA/AG/GG)

$62.6 / 33.5 / 3.9$

$71.1 / 25.5 / 3.3$

0.002

Data are shown as mean \pm standard deviation, median [interquartile range] or percentage. * $S$ tudent's $t$ test, Mann Whitney's U test or Chi square test. HDL: High density lipoprotein, LDL: low density lipoprotein.

\section{Association Of Polymorphisms And Haplotypes With Hypoalphalipoproteinemia}

All the SNPs were in Hardy-Weinberg equilibrium. Figure 1 shows the associated DPP4 polymorphisms with HA. For this analysis, we adjusted each model for age, sex, BMI, VAF, current smoking, physical activity, and triglycerides levels. Under heterozygote model the rs 12617336 polymorphism (0.22 [0.09$0.54] \mathrm{P}=0.001$ ) was significantly associated with a low risk of HA. Additionally, the rs 17574 polymorphism was also associated with protection for the presence of $\mathrm{HA}$, under additive $(0.79$ [0.64$0.98] \mathrm{P}=0.033)$ dominant $(0.75[0.58-0.96] \mathrm{P}=0.021)$, heterozygote $(0.75[0.58-0.97] \mathrm{P}=0.029)$ and codominant $1(0.74[0.57-0.96] \mathrm{P}=0.024)$ models.

The five studied SNPs were in linkage disequilibrium ( $\left.D^{\prime}>0.75\right)$ and nine different haplotypes were constructed (GTTCA, GCCTA, GTCCA, GTTCG, GCCCA, GTCCG, GTCTA, GCCTG and GCCCG). The GCCTA haplotype was associated to $21 \%$ higher risk to present HA (1.210 [1.023-1.431], $\mathrm{P}=0.026)$; in contrast, the GTTCG haplotype was related to $25 \%$ lower risk of HA $(0.749$ [0.586-0.957] P $=0.021)$ (Table 2$)$. 
Table 2

DPP4 haplotype frequencies and the presence of hypoalphalipoproteinemia.

\begin{tabular}{|c|c|c|c|c|c|}
\hline \multirow[t]{2}{*}{ Haplotypes } & & \multicolumn{2}{|c|}{ Hypoalphalipoproteinemia } & \multirow[t]{2}{*}{ OR [95\% Cl] } & \multirow[t]{2}{*}{$P$ value } \\
\hline & & Yes & No & & \\
\hline $\mathrm{H} 1$ & GTTCA & 0.328 & 0.321 & $1.035[0.888-1.208]$ & 0.658 \\
\hline $\mathrm{H} 2$ & GCCTA & 0.264 & 0.229 & $1.210[1.023-1.431]$ & 0.026 \\
\hline $\mathrm{H} 3$ & GTCCA & 0.119 & 0.107 & $1.139[0.907-1.430]$ & 0.264 \\
\hline $\mathrm{H} 4$ & GTTCG & 0.084 & 0.110 & $0.749[0.586-0.957]$ & 0.021 \\
\hline H5 & GCCCA & 0.083 & 0.091 & $0.905[0.701-1.169]$ & 0.445 \\
\hline $\mathrm{H} 6$ & GTCCG & 0.031 & 0.032 & $0.956[0.633-1.446]$ & 0.832 \\
\hline $\mathrm{H} 7$ & GTCTA & 0.022 & 0.024 & $0.921[0.569-1.490]$ & 0.737 \\
\hline $\mathrm{H} 8$ & GCCTG & 0.019 & 0.026 & $0.697[0.426-1.139]$ & 0.149 \\
\hline $\mathrm{H} 9$ & GCCCG & 0.017 & 0.020 & $0.845[0.498-1.437]$ & 0.535 \\
\hline
\end{tabular}

Cardiovascular risk factors and their relation with rs12617336 and rs17574 DPP4 polymorphisms.

Table 3 shows the associations of DPP4 rs 12617336 and rs17574 polymorphisms with cardiovascular risk factors in individuals with and without HA. In HA individuals, under heterozygote model, the rs12617336 was associated with lower risk of $I R(O R=0.169, P=0.019)$ and $H I(O R=0.108, P=0.006)$, and with 6 times higher risk of hyperuricemia $(O R=6.296, P=0.012)$. On the other hand, in the non-HA subjects, the rs 17574 was related with $25 \%$ lower risk of $I R\left(O R=0.751, P_{\text {additive }}=0.049\right)$, approximately $40 \%$ higher risk of high non-HDL-C $\left(O R=1.478, P_{\text {heterozygote }}=0.023 ; \mathrm{OR}=1.447, \mathrm{P}_{\text {co-dominant } 1}=0.033 ;\right)$ and high apoB $\left(O R=1.438, P_{\text {heterozygote }}=0.043\right)($ Table 3$)$. 
Table 3

DDP4 gene polymorphisms associated with cardiovascular risk factors.

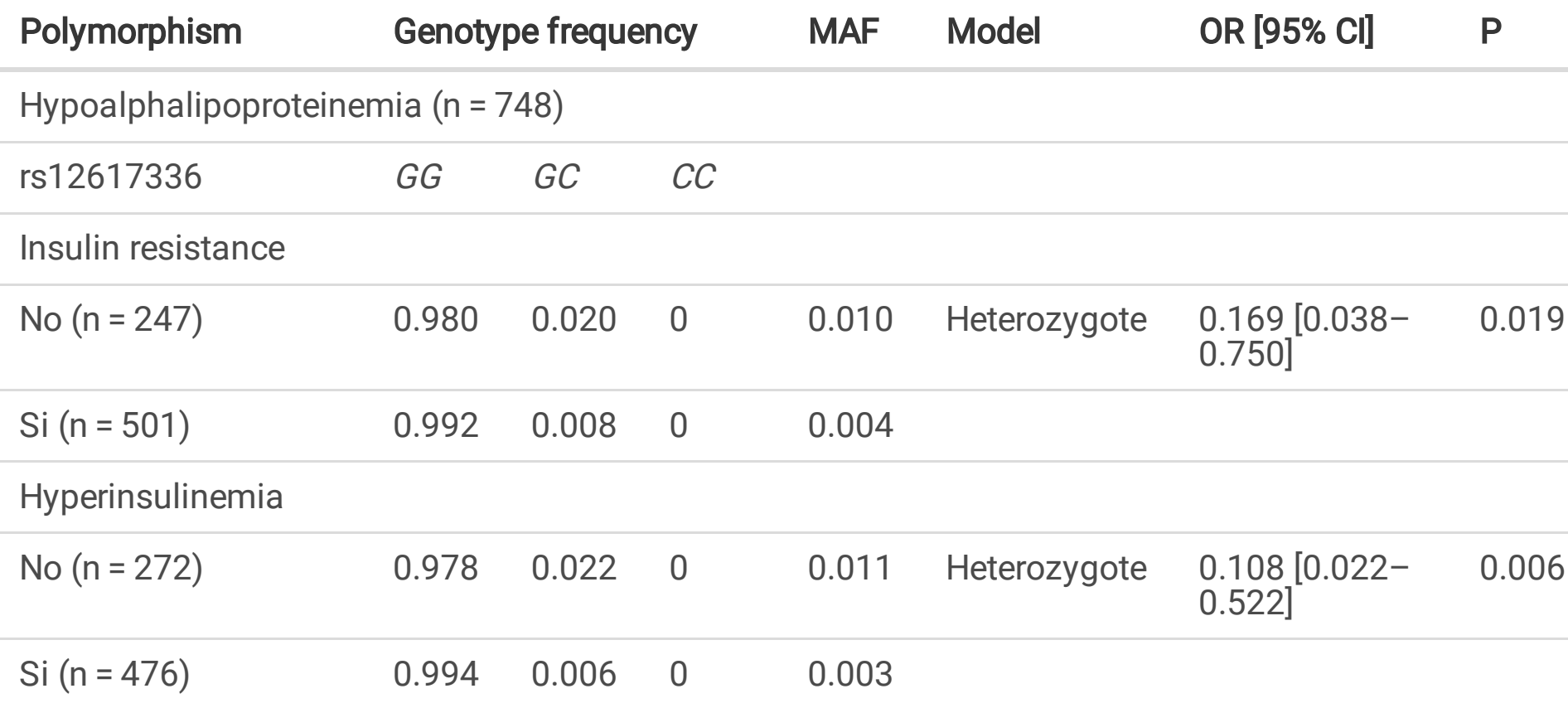

Hyperuricemia

$\begin{array}{lllllll}\text { No }(n=557) & 0.995 & 0.005 & 0 & 0.003 & \text { Heterozygote } & \left.\begin{array}{l}6.296[1.505- \\ 26.34\end{array}\right]\end{array}$

$\begin{array}{lllll}\text { Si }(n=191) & 0.969 & 0.031 & 0 & 0.016\end{array}$

Non-hypoalphalipoproteinemia $(\mathrm{n}=$

745)

rs17574 $\quad A A \quad A G \quad G G$

Insulin resistance

$\begin{array}{llllllll}\text { No }(\mathrm{n}=389) & 0.588 & 0.366 & 0.046 & 0.229 & \text { Additive } & \begin{array}{l}0.751[0.564- \\ 0.999\end{array}\end{array}$

Si $(n=356) \quad 0.668 \quad 0.301 \quad 0.031 \quad 0.181$

Non-HDL-C >

$160 \mathrm{mg} / \mathrm{dL}$

$\begin{array}{llllllll}\text { No }(n=530) & 0.643 & 0.314 & 0.043 & 0.200 & \text { Heterozygote } & 1.478[1.055- & 0.023 \\ & & & & & & 2.072\end{array}$

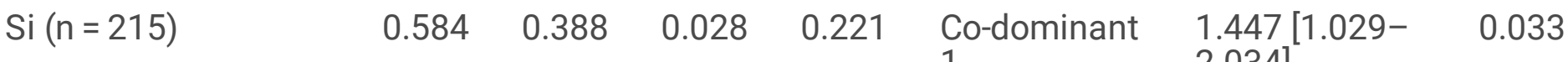

High apolipoprotein

B

MAF, minor allele frequency; OR, odds ratio; $95 \% \mathrm{Cl}, 95 \%$ confidence interval; $\mathrm{p} 75$, percentile 75 . All models were adjusted by age, gender, and body mass index. 


\begin{tabular}{|c|c|c|c|c|c|c|c|}
\hline Polymorphism & Genoty & e freque & ncy & MAF & Model & OR $[95 \% \mathrm{Cl}]$ & $P$ \\
\hline No $(n=551)$ & 0.636 & 0.320 & 0.044 & 0.203 & Heterozygote & $\begin{array}{l}1.438 \text { [1.011- } \\
1.045]\end{array}$ & 0.043 \\
\hline Si $(n=194)$ & 0.589 & 0.385 & 0.026 & 0.219 & & & \\
\hline
\end{tabular}

\section{Relationship between DPP4 genotypes and DPP4 serum concentrations}

Figure 2A shows that DPP4 serum concentration was higher in the non-HA subjects when compared with HA participants (125.2 [98.4-153.7] ng/mL vs 117.8 [94.6-148.9] ng/mL, $\mathrm{P}=0.008$, respectively). In the same way, the DPP4 concentration of the whole sample was analyzed stratifying for rs 17574 DPP4 genotypes. Carriers of the rs 17574AA genotype have the highest DPP4 serum concentration (123.3 [97.3$155.1] \mathrm{ng} / \mathrm{mL}$ ) compared with $A G$ (120.6 [93.7-145.2] $\mathrm{ng} / \mathrm{mL})$ and $G G$ genotypes (116.5 [86.4-142.1] $\mathrm{ng} / \mathrm{mL}, \mathrm{P}=0.038$ ) (Fig. 2B), and compared to carriers of $G$ allele ( $A G$ and $G G$ genotypes) (120.3 [92.8.144] $\mathrm{ng} / \mathrm{mL}, \mathrm{P}=0.014$ vs $A A$ genotype) (Fig. $2 \mathrm{C}$ ).

The DPP4 serum concentration and its association with rs17574 genotypes were evaluated separately in individuals with and without HA (Fig. 3). In individuals without HA no significant differences where observed (Figs. $3 \mathrm{~A}$ and $3 \mathrm{~B}$ ). On the other hand, in individuals with $\mathrm{HA}$, subjects with $A A$ genotype had the highest DPP4 concentration (119 [95.9-152.4] ng/mL) compared with $A G(113.5[92.0-142.9] \mathrm{ng} / \mathrm{mL}$ ) and GG genotypes (114.6 [80.9-142.1], $\mathrm{P}=0.042)(\mathrm{Fig} .3 \mathrm{C}$ ); and also, when compared with carriers of $G$ allele ( $A G$ and $G G$ genotypes) (113.8 [91.4-142.5] ng/mL, $\mathrm{P}=0.013$ vs. $A A$ genotype) (Fig. 3D). Due to the lack of the presence of the $C C$ genotype of the SNP rs12617336 in our population, we were unable to assess the association of the genotypes of this SNP with DPP4 concentrations.

\section{Discussion}

To the best of our knowledge, this report shows the association of rs12617336 and rs17574 DPP4 polymorphisms with HA for the first time. The polymorphisms were also associated with IR (rs12617336 and rs17574), HI (rs12617336), hyperuricemia (rs12617336), high non-HDL-C levels (rs17574) and high apoB levels (rs17574). When compared with non-HA individuals, lower DPP4 levels were observed in the HA group. Differences in DPP4 levels were observed among rs 17574 genotypes, with the highest levels in rs17574 AA genotype individuals. Thus, individuals with the $G$ allele presented low levels of DPP4, which agree with the protective effect of this allele.

Adipocytes secret several active proteins called adipokines and it is well-known that the deregulation of their secretion is associated with metabolic diseases[35]. DPP4 is expressed in adipocytes, and in patients with obesity, its expression and activity are elevated[36]. DPP4 plasma levels are related to several markers of obesity, such as BMI, waist circumference, plasma triglyceride levels, leptin concentration, and fat cell volume[10]. In this report, the rs12617336 and rs17574 were associated with a 
$20-25 \%$ risk reduction for the presence of $\mathrm{HA}$, a metabolic abnormality that often coexists with obesity. Moreover, the GTTCG haplotype that contains the rs $17574 \mathrm{G}$ protector allele was also related to reduced risk (-25\%) of this condition. Neither of the polymorphisms, rs 17574 or rs 12617336 , has been previously associated with HA. Turcot et.al. reported that individuals with rs 17574 GG genotype had higher levels of methylation in the promote $\mathrm{CpG}$ islands in the visceral adipose tissue[37]. They also showed a positive relationship between methylation levels and HDL-C concentration[37]. Additionally, using informatics tools, important possible functional effects were defined for the two polymorphisms associated with HA. The rs 17574 polymorphism, that we report associated with a low risk of HA, is located in exon 2 of the gene; the computer analysis that we carried out establishes that the $G$ allele in this position can affect the efficiency of cutting and splicing, modifying the binding affinity for splicing factors SF2ASF1 and SF2ASF2. This suggests that this polymorphism could be related to the production of DPP4 isoforms with altered activity. Previously, various forms of DPP4 were detected in human and placental plasma[38, 39], as well as in lung cancer tissue and normal tissue[40, 41]. DPP4 isoforms with altered activity could be involved in the development of HA. On the other hand, the rs $12617336 \mathrm{G}$ allele produces a binding site for the miRNA-939, miRNA-708, and miRNA-1244, whereas the $C$ allele produces a binding site for the miRNA-1238. It has also been reported that HDLs transport miRNAs[42, 43], some of them regulate crucial pathways for maintaining cardiovascular homeostasis[44] and protect against atherosclerosis by modulating HDL cholesterol efflux capacity and lipid metabolism[45, 46]. These data agree with our results showing that the rs12617336 and rs17574 polymorphisms are associated with a reduced risk for HA. Our findings suggest that rs $12617336 C$ and rs $17574 \mathrm{G}$ alleles could be considered as potential genetic markers for HA in our population.

DPP4 enzymatic activity inactivates GLP-1, a hormone that regulates postprandial insulin secretion[47]. Therefore, DPP4 increased level and/or activity may impair insulin sensitivity causing IR and HI. It has been reported that DPP4 serum levels were higher in IR as compared to insulin-sensitive subjects matched for BMI[16]. Moreover, in T2DM it has been shown that increased DPP4 activity is positively associated with IR with a significant increase in IR with rising DPP4 activity quartiles[15]. In the same way, it has been reported that increase DPP4 mRNA levels in the liver of patients with non-alcoholic FL disease correlate with IR.[17]. It has also been reported that DPP4 expression correlates with the amount of VAF, adipocyte size, and inflammation[16]. Macrophages and dendritic cells present in VAF exhibit increased DPP4 expression in response to obese state or inflammation[48]. The best-known non-catalytic function DPP4 exerts is the co-stimulation of T cells through interaction with adenosine deaminase[49] and modulation of the function of antigen-presenting cells[18]. This suggests that DPP4 could have an important participation in the chronic low-grade inflammation present in dyslipidemias, T2DM, IR, obesity, and atherosclerosis. When chronic inflammation is present in liver and adipose tissue, the activated resident macrophages release pro-inflammatory cytokines; by a direct interaction between inflammatory pathways and insulin signaling, these cytokines can cause IR,[50] and in consequently HI. These data support our findings that showed a significant association between the rs17574 and rs12617336 polymorphisms with a low risk for IR and HI (rs12617336). In our study, the carriers of the protective 
genotype rs17574GG showed the lowest levels of serum DPP4, suggesting that lower DPP4 activity in these subjects would result in higher incretin levels and insulin sensitivity.

Of the five DPP4 polymorphisms included in the present study, three of them have previously been associated with serum lipid levels[51,52] and apoB levels[21]. Among T2DM individuals, dyslipidemia is a major risk factor for CVD[53]. ApoB plasma levels, the principal protein component of very-low-density lipoprotein $(V L D L)$ remanent, VLDL, and LDL particles, represent the total atherogenic lipoprotein particles in circulation and correlates with the concentration of non-HDL-C[54]. In South Asians, Bayle et al[21] identified and replicated the association between variation at the rs4664443 DPP4 polymorphism and apoB levels. They also report an interaction between the SNP and BMI for plasma apoB levels in Europeans[21]. On the other hand, the DPP4 rs 1558957 has been previously associated with high total cholesterol, HDL-C, LDL-C, and triglycerides plasma concentrations in a study of obese individuals of European ancestry, although these results were inconsistent[52]. These previous reports support our findings. Here, we show an association between rs17574 and the presence of high apoB and non-HDL-C levels. Consistent with the observed association between polymorphisms in the DPP4 gene with apoB and non-HDL-C, pharmacological inhibition of DPP4, has been found associated with lower total cholesterol, apoB, triglycerides, non-HDL-C and VLDL levels[55-57].

Uric acid, the product of purine catabolism, is a natural antioxidant. However, it is well recognized that hyperuricemia is associated with metabolic diseases and endothelial dysfunction. Consistent with our observed association between variation in DPP4 polymorphisms and hyperuricemia, Mohandas et al. demonstrated that uric acid inhibits DPP4 activity when it is anchored to the membrane and that the inhibitory effect depends on the redox state of cells and formation of intracellular triuret[58].

DPP4 is ubiquitously expressed on the surface of several cell types, however, it can also be found in circulation after it is shed from the membrane by proteolytic cleavage[59]. Increased DPP4 circulatory levels, in metabolic diseases, could be explained by an aberrant DPP4 shedding. It has been reported that in smooth muscle cells and adipocytes, hypoxia increased DPP4 shedding by matrix metalloproteases[59]. The constitutive shedding mechanism of DPP4 is varied with cell specificity as well as cells and tissues circumstances and occurs due to a complex interplay between different proteases in cell type-specific manner[60]. Our results showed that non-HA subjects have significantly higher DPP4 concentrations. These findings appear to be contradictory to those expected. However, epigenetic modifications and changes at the DNA level are part of the complex mechanisms that modulate the production of several molecules, including DPP4. As previously mentioned, Turcot et al. in 2011 reported in premenopausal obese nondiabetic women that carriers of the rs17574 GG genotype have higher levels of methylation[37]. Our findings show that carriers of the rs $17574 \mathrm{GG}$ genotype have significantly lower concentrations compared to $A G$ and $A A$ genotypes. When stratified by the presence of $\mathrm{HA}$, the statistically significant difference was maintained in the subjects with HA. Individuals with HA, have a higher amount of VAF in comparison with non-HA $\left(159 \mathrm{~cm}^{2}\right.$ vs $134 \mathrm{~cm}^{2}$, respectively, $\left.\mathrm{p}<0.001\right)$. The aforementioned suggests that in this group, the presence of higher VAF could be associated with an increase in the 
percentage of methylation in carriers of the rs17574 GG genotype, and thus, lead to lower expression of the DPP4 gene and, therefore, to lower levels in protein plasma.

The main strength of the present work is the inclusion of a large cohort of Mexican individuals, with and without HA, who were broadly characterized from a tomographic, clinical, and biochemical point of view using standardized methods, which allowed adjust the results by an important number of potential confounders. Although the selection of the participants was not random, the studied subjects were not aware of being carriers of the variants of the DPP4 gene polymorphisms studied, given this, it could be expected that the observed associations were similar to those of a sample random and therefore be applied to the general population. Despite these strengths, our study has some limitations. First, due to the cross-sectional nature of the study, we cannot make causal conclusions. Second, our results were not replicated in the second cohort of individuals with and without HA. Thirdly, IR was not determined by the gold standard: the euglycemic clamp; nevertheless, insulin sensitivity can be accurately estimated by the homeostasis model assessment of insulin resistance (HOMA-IR) index that has proven to be a reliable measure[61]. Fourthly, our data may not apply to other ethnicities, considering that the GEA participants are exclusively Mexican-Mestizo subjects. The DPP4 polymorphism associations detected in our study should be investigated in other populations to establish if they are shared with other ethnic groups or are specific for the Mexican population.

\section{Conclusions}

In summary, our results suggest that the rs 12617336 and rs17574 polymorphisms, as genotypes and haplotypes, are associated with a low risk to present HA. The polymorphisms were also associated with protection for the presence of IR and HI. Additionally, we found an association with hyperuricemia, high levels of non-HDL-C, and apoB. DPP4 levels were higher in non-HA individuals when compared to HA individuals. Individuals with rs $17574 A A$ genotype presented higher levels of DPP4 when compared to individuals with other genotypes. Considering that this is the first study to report the association of DPP4 polymorphisms with the presence of HA, studies in other populations are necessary to confirm our results.

\section{Abbreviations}

apoB

Apolipoprotein B; BMI:Body mass index; CVD:Cardiovascular disease; CAC:Coronary artery calcification; Cl:Confidence intervals; DPP4; Dipeptidyl peptidase-4; FL:Fatty liver; GEA:Genetics of Atherosclerotic Disease; GIP; Glucose-dependent insulinotropic polypeptide; GLP-1:Glucagon-like peptide-1; HDL:Highdensity lipoprotein; HDL-C:High-density lipoprotein cholesterol; HI:Hyperinsulinemia; HA:Hypoalphalipoproteinemia; hsCRP:high sensitivity $\mathrm{C}$ reactive protein; HOMA:Homeostasis model assessment of insulin resistance; IR:Insulin resistance; LDL:Low-density lipoprotein; MAF:Minor allele frequency; non-HDL-C:non-HDL-cholesterol; OR:Odds ratio; PCAD:Premature coronary artery disease; 
SA:Subclinical atherosclerosis; SNPs:Single nucleotide polymorphisms; T2DM:Type 2 diabetes mellitus; VAF:Visceral adipose fat; VLDL:Very low-density lipoprotein.

\section{Declarations}

\section{Acknowledgments}

The authors are grateful to María Oliva Romero-Arroyo for her technical assistance.

\section{Authors' contributions}

All the authors have contributed significantly. Conceptualization, R.P-S., and G.V.-A.; Methodology, F.S.-M., J.R.-B., C.V.-V. and A.H.-DC.; Formal analysis, C.O.-Y. and M.S.-G.; Resources, G.V.-A. and R.P.-S.; Visualization, M.d.C.S.-G., C.O.-Y. and M.S.-G.; Supervision, S.A.C.-V. and M.d.C.G.-S.; Project administration, M.d.C.G.-S., S.A.C.-V., G.V.-A. and R.P.-S.; Funding acquisition, R.P.-S.; Data curation, M.d.C.S.-G, S.A.C.-V., J.R.-B., C.V.-V., A.H.-DC. and F.S.-M.; Writing-original draft preparation, R.P.-S., G.V.-A., S.A.C.-V., and M.d.C.G.-S.; Writing-review and editing, G.V.-A. and R.P.-S. All authors have read and agreed to the published version of the manuscript.

\section{Funding}

This project was funded by CONSEJO NACIONAL DE CIENCIA Y TECNOLOGÍA (Grant number: Ciencia Básica 2016-01-286065).

\section{Availability of data and materials}

All analyzed and derivative raw data are available on request.

\section{Ethics approval and consent to participate}

The study was conducted in accordance with the Declaration of Helsinki and was approved by the Ethics Committee of the Instituto Nacional de Cardiología Ignacio Chávez (Project number 18-1082). All participants gave their written informed consent for inclusion before they participated in the study.

\section{Consent for publication}

Not applicable

\section{Competing interests}

The authors declare that they have no competing interests

\section{References}


1. Chapman MJ, Ginsberg HN, Amarenco P, et al. Triglyceride-rich lipoproteins and high-density lipoprotein cholesterol in patients at high risk of cardiovascular disease: evidence and guidance for management. Eur Heart J. 2011;32:1345-61. https://doi.org/10.1093/eurheartj/ehr112.

2. Ponte-Negretti $\mathrm{Cl}$, Isea-Perez JE, Lorenzatti AJ, et al. Atherogenic Dyslipidemia in Latin America: Prevalence, causes and treatment. Int J Cardiol. 2017;243:516-22. https://doi.org/10.1016/j.ijcard.2017.05.059.

3. Hernández-Alcaraz C, Aguilar-Salinas CA, Mendoza-Herrera K, et al. Dyslipidemia prevalence, awareness, treatment and control in Mexico: results of the Ensanut 2012. Salud Pública Mex. 2020;62:137-46. https://doi.org/10.21149/10520.

4. Goff D, Lloyd-Jones D, Bennett G, et al. 2013 ACC/AHA Guideline on the Assessment of Cardiovascular Risk: A Report of the American College of Cardiology/American Heart Association Task Force on Practice Guidelines. Circulation. 2014;129:49-73. https://doi.org/10.1161/01.CIR.0000437741.48606.98.

5. Ingelsson E, Schaefer E, Contois J, et al. Clinical Utility of Different Lipid Measures for Prediction of Coronary Heart Disease in Men and Women. JAMA. 2007;298:776-85. https://doi.org/10.1001/JAMA.298.7.776.

6. Brousseau ME, Goldkamp AL, Collins D, et al. Polymorphisms in the gene encoding lipoprotein lipase in men with low HDL-C and coronary heart disease. J Lipid Res. 2004;45:1885-91. https://doi.org/10.1194/jlr.M400152-JLR200.

7. Brousseau ME, Bodzioch M, Schaefer EJ, et al. Common variants in the gene encoding ATP-binding cassette transporter 1 in men with low HDL cholesterol levels and coronary heart disease. Atherosclerosis. 2001;154:607-11. https://doi.org/10.1016/S0021-9150(00)00722-X.

8. Brousseau ME, O'Connor JJ, Ordovas JM, et al. Cholesteryl Ester Transfer Protein Taq I B2B2 Genotype Is Associated With Higher HDL Cholesterol Levels and Lower Risk of Coronary Heart Disease End Points in Men With HDL Deficiency. Arterioscler Thromb Vasc Biol. 2002;22:1148-54. https://doi.org/10.1161/01.ATV.0000024566.57589.2E.

9. Mulvihill EE, Drucker DJ. Pharmacology, Physiology, and Mechanisms of Action of Dipeptidyl Peptidase-4 Inhibitors. Endocr Rev. 2014;35:992-1019. https://doi.org/10.1210/er.2014-1035.

10. Lamers D, Famulla S, Wronkowitz N, et al. Dipeptidyl Peptidase 4 Is a Novel Adipokine Potentially Linking Obesity to the Metabolic Syndrome. Diabetes. 2011;60:1917-25. https://doi.org/10.2337/db10-1707.

11. Zhao T, Parikh P, Bhashyam S, et al. Direct effects of glucagon-like peptide-1 on myocardial contractility and glucose uptake in normal and postischemic isolated rat hearts. J Pharmacol Exp Ther. 2006;317:1106-13. https://doi.org/10.1124/jpet.106.100982.

12. Zheng T, Gao Y, Baskota A, Chen T, Ran X, Tian H. Increased plasma DPP4 activity is predictive of prediabetes and type 2 diabetes onset in Chinese over a four-year period: result from the China National Diabetes and Metabolic Disorders Study. J Clin Endocrinol Metab. 2014;99:E2330-4. https://doi.org/10.1210/jc.2014-1480. 
13. Zheng T, Chen T, Liu Y, Gao Y, Tian H. Increased plasma DPP4 activity predicts new-onset hypertension in Chinese over a 4-year period: possible associations with inflammation and oxidative stress. J Hum Hypertens. 2015;29:424-9. https://doi.org/10.1038/jhh.2014.111.

14. Baumeier C, Saussenthaler S, Kammel A, et al. Hepatic DPP4 DNA Methylation Associates With Fatty Liver. Diabetes. 2017;66:25-35. https://doi.org/10.2337/db15-1716.

15. Zheng TP, Liu YH, Yang LX, Qin SH, Liu HB. Increased plasma dipeptidyl peptidase-4 activities are associated with high prevalence of subclinical atherosclerosis in Chinese patients with newly diagnosed type 2 diabetes: a cross-sectional study. Atherosclerosis. 2015;242:580-8. https://doi.org/10.1016/j.atherosclerosis.2015.07.042.

16. Sell H, Blüher M, Klöting N, et al. Adipose dipeptidyl peptidase-4 and obesity: correlation with insulin resistance and depot-specific release from adipose tissue in vivo and in vitro. Diabetes Care. 2013;36:4083-90. https://doi.org/10.2337/dc13-0496.

17. Miyazaki M, Kato M, Tanaka K, et al. Increased hepatic expression of dipeptidyl peptidase-4 in nonalcoholic fatty liver disease and its association with insulin resistance and glucose metabolism. Mol Med Rep. 2012:5:729-733. https://doi.org/10.3892/mmr.2011.707.

18. Zhong J, Gong Q, Goud A, Srinivasamaharaj S, Rajagopalan S. Recent advances in dipeptidylpeptidase-4 inhibition therapy: Lessons from the bench and clinical trials. J Diabetes Res. 2015;2015:606031. https://doi.org/10.1155/2015/606031.

19. Jiang L, Yin J, Ye L, et al. Novel risk loci for rheumatoid arthritis in Han Chinese and congruence with risk variants in Europeans. Arthritis Rheumatol. 2014;66:1121-32. https://doi.org/10.1002/art.38353.

20. Ahmed RH, Huri HZ, Al-Hamodi Z, Salem SD, Al-Absi B, Muniandy S. Association of DPP4 gene polymorphisms with type 2 diabetes mellitus in Malaysian subjects. PLoS ONE. 2016;11:1-12. https://doi.org/10.1371/journal.pone.0154369.

21. Bailey SD, Xie C, Paré G, et al. Variation at the DPP4 locus influences apolipoprotein B levels in South Asians and exhibits heterogeneity in Europeans related to BMI. Diabetologia. 2014;57:738-45. https://doi.org/10.1007/s00125-013-3142-3.

22. Aghili N, Devaney JM, Alderman LO, et al. Polymorphisms in dipeptidyl peptidase IV gene are associated with the risk of myocardial infarction in patients with atherosclerosis. Neuropeptides. 2012;46:367-71. https://doi.org/10.1016/j.npep.2012.10.001.

23. Rtveladze K, Marsh T, Barquera S, et al. Obesity prevalence in Mexico: impact on health and economic burden. Public Health Nutr. 2014;17:233-9. https://doi.org/10.1017/S1368980013000086.

24. Medina-Urrutia A, Posadas-Romero C, Posadas-Sánchez R, et al. Role of adiponectin and free fatty acids on the association between abdominal visceral fat and insulin resistance. Cardiovasc Diabetol. 2015;14:20. https://doi.org/10.1186/s12933-015-0184-5.

25. Posadas-Sánchez R, López-Uribe AR, Posadas-Romero C, et al. Association of the I148M/PNPLA3 (rs738409) polymorphism with premature coronary artery disease, fatty liver, and insulin resistance 
in type 2 diabetic patients and healthy controls. The GEA study. Immunobiology. 2017;222:960-6. https://doi.org/10.1016/j.imbio.2016.08.008.

26. Posadas-Sánchez R, Pérez-Hernández N, Angeles-Martínez J, et al. Interleukin 35 Polymorphisms Are Associated with Decreased Risk of Premature Coronary Artery Disease, Metabolic Parameters, and IL35 Levels: The Genetics of Atherosclerotic Disease (GEA) Study. Mediators Inflamm. 2017;2017:1 10. https://doi.org/10.1155/2017/6012795.

27. Grundy SM, Cleeman JI, Daniels SR, et al. American Heart Association, National Heart, Lung, and Blood Institute, Diagnosis and Management of the Metabolic Syndrome: An American Heart Association/National Heart, Lung, and Blood Institute Scientific Statement. Circulation. 2005;112:2735-52. https://doi.org/10.1161/CIRCULATIONAHA.105.169404.

28. Grundy SM. Low-Density, Lipoprotein. Non-High-Density Lipoprotein, and Apolipoprotein B as Targets of Lipid-Lowering Therapy. Circulation. 2002;106:2526-9. https://doi.org/10.1161/01.CIR.0000038419.53000.D6.

29. Mazer N, Giulianini F, Paynter N, Jordan P, Mora S. A Comparison of the Theoretical Relationship Between HDL Size and the Ratio of HDL Cholesterol to Apolipoprotein A-I With Experimental Results From the Women's Health Study. Clin Chem. 2013;59:949-58.

https://doi.org/10.1373/CLINCHEM.2012.196949.

30. Hirano T, Ito Y, Yoshino G. Measurement of small dense low-density lipoprotein particles. J Atheroscler Thromb. 2005;12:67-72.

31. Ogbera AO, Azenabor AO. Hyperuricaemia and the metabolic syndrome in type $2 \mathrm{DM}$. Diabetol Metab Syndr. 2010;2:24. https://doi.org/10.1186/1758-5996-2-24.

32. Kvist H, Chowdhury B, Grangård U, Tylén U, Sjöström L. Total and visceral adipose-tissue volumes derived from measurements with computed tomography in adult men and women: predictive equations. Am J Clin Nutr. 1988;48:1351-61.

33. Mautner GC, Mautner SL, Froehlich J, et al. Coronary artery calcification: assessment with electron beam CT and histomorphometric correlation. Radiology. 1994;192:619-23. https://doi.org/10.1148/radiology.192.3.8058924.

34. Baecke JA, Burema J, Frijters JE. A short questionnaire for the measurement of habitual physical activity in epidemiological studies. Am J Clin Nutr. 1982;36:936-42. https://doi.org/10.1093/ajcn/36.5.936.

35. Wellen KE, Hotamisligil GS. Inflammation, stress, and diabetes. J Clin Invest. 2005;115:1111-9. https://doi.org/10.1172/JCl25102.

36. Stengel $A$, Goebel-Stengel $M$, Teuffel $P$, et al. Obese patients have higher circulating protein levels of dipeptidyl peptidase IV. Peptides. 2014;61:75-82. https://doi.org/10.1016/j.peptides.2014.09.006.

37. Turcot V, Bouchard L, Faucher G, et al. DPP4 gene DNA methylation in the omentum is associated with its gene expression and plasma lipid profile in severe obesity. Obesity. 2011;19:388-95. https://doi.org/10.1038/oby.2010.198. 
38. Krepela E, Kraml J, Vicar J, Kadlecová L, Kasafírek E. Demonstration of two molecular forms of dipeptidyl peptidase IV in normal human serum. Physiol Bohemoslov. 1983;32:486-96.

39. Puschel G, Mentlein R, Heymann E. Isolation and Characterization of Dipeptidyl Peptidase IV from Human Placenta. Eur J Biochem. 1982;126:359-65. https://doi.org/10.1111/j.14321033.1982.tb06788.x.

40. Sedo A, Krepela E, Kasafírek E, Kraml J, Kadlecová L. Dipeptidyl peptidase IV in the human lung and spinocellular lung cancer. Physiol Res. 1991;40:359-62.

41. Sedo A, Malík R, Krepela E. Dipeptidyl peptidase IV in C6 rat glioma cell line differentiation. Biol Chem. 1998;379:39-44.

42. Ren J, Zhang J, Xu N, et al. Signature of Circulating microRNAs as Potential Biomarkers in Vulnerable Coronary Artery Disease. PloS One. 2013;8:e80738. https://doi.org/10.1371/JOURNAL.PONE.0080738.

43. Wagner J, Riwanto M, Besler C, et al. Characterization of Levels and Cellular Transfer of Circulating Lipoprotein-Bound microRNAs. Arterioscler Thromb Vasc Biol. 2013;33:1392-400. https://doi.org/10.1161/ATVBAHA.112.300741.

44. Vickers K, Palmisano B, Shoucri B, Shamburek R, Remaley A. MicroRNAs Are Transported in Plasma and Delivered to Recipient Cells by High-Density Lipoproteins. Nat Cell Biol. 2011;13:423-33. https://doi.org/10.1038/NCB2210.

45. Vickers K, Landstreet S, Levin M, et al. MicroRNA-223 Coordinates Cholesterol Homeostasis. Proc Natl Acad Sci USA. 2014;111:14518-23. https://doi.org/10.1073/PNAS.1215767111.

46. Ono K. Functions of microRNA-33a/b and microRNA Therapeutics. J Cardiol. 2016;67:28-33. https://doi.org/10.1016/J.JJCC.2015.10.017.

47. Gorrell MD. Dipeptidyl peptidase IV and related enzymes in cell biology and liver disorders. Clin Sci (Lond). 2005;108:277-92. https://doi.org/10.1042/CS20040302.

48. Zhong J, Rao X, Deiuliis J, et al. A Potential Role for Dendritic Cell/Macrophage-Expressing DPP4 in Obesity-Induced Visceral Inflammation. Diabetes. 2013;62:149-57. https://doi.org/10.2337/DB120230.

49. Kameoka J, Tanaka T, Nojima Y, Schlossman SF, Morimoto C. Direct association of adenosine deaminase with a T cell activation antigen, CD26. Science. 1993;261:466-9.

50. de Luca C, Olefsky JM. Inflammation and insulin resistance. FEBS Lett. 2008;582:97-105. https://doi.org/10.1016/j.febslet.2007.11.057.

51. Xing X, Han Y, Zhou X, et al. Association between DPP4 gene polymorphism and serum lipid levels in Chinese type 2 diabetes individuals. Neuropeptides. 2016;60:1-6. https://doi.org/10.1016/j.npep.2016.08.005.

52. Bouchard L, Faucher G, Tchernof A, et al. Comprehensive genetic analysis of the dipeptidyl peptidase-4 gene and cardiovascular disease risk factors in obese individuals. Acta Diabetol. 2009;46:13-21. https://doi.org/10.1038/oby.2010.198. 
53. Mooradian A. Dyslipidemia in Type 2 Diabetes Mellitus, Nature Clinical Practice. Endocrinol Metab. 2009;5:150-9. https://doi.org/10.1038/NCPENDMET1066.

54. Pischon T, Girman C, Sacks F, Rifai N, Stampfer M, Rimm E. Non-high-density Lipoprotein Cholesterol and Apolipoprotein B in the Prediction of Coronary Heart Disease in Men. Circulation. 2005;112:3375-83. https://doi.org/10.1161/CIRCULATIONAHA.104.532499.

55. Monami M, Lamanna C, Desideri C, Mannucci E. DPP-4 Inhibitors and Lipids: Systematic Review and Meta-Analysis. Adv Ther. 2012;29:14-25. https://doi.org/10.1007/S12325-011-0088-Z.

56. Tremblay A, Lamarche B, Deacon C, Weisnagel S, Couture P. Effect of Sitagliptin Therapy on Postprandial Lipoprotein Levels in Patients With Type 2 Diabetes. Diabetes Obes Metab. 2011;13:366-73. https://doi.org/10.1111/J.1463-1326.2011.01362.X.

57. Kim J, Kim S, Baek H, et al. Comparison of Vildagliptin and Pioglitazone in Korean Patients With Type 2 Diabetes Inadequately Controlled With Metformin. Diabetes Metab J. 2016;40:230-9. https://doi.org/10.4093/DMJ.2016.40.3.230.

58. Mohandas R, Sautina L, Beem E, et al. Uric Acid Inhibition of Dipeptidyl Peptidase IV in Vitro Is Dependent on the Intracellular Formation of Triuret. Exp Cell Res. 2014;326:136-42. https://doi.org/10.1016/J.YEXCR.2014.05.025.

59. Röhrborn D, Eckel J, Sell H. Shedding of Dipeptidyl Peptidase 4 Is Mediated by Metalloproteases and Up-Regulated by Hypoxia in Human Adipocytes and Smooth Muscle Cells. FEBS Lett. 2014;588:3870-7. https://doi.org/10.1016/J.FEBSLET.2014.08.029.

60. Nargis T, Chakrabarti P. Significance of circulatory DPP4 activity in metabolic diseases. IUBMB Life. 2018;70:112-9. https://doi.org/10.1002/iub.1709.

61. Bonora E, Targher G, Alberiche M, et al. Homeostasis model assessment closely mirrors the glucose clamp technique in the assessment of insulin sensitivity: studies in subjects with various degrees of glucose tolerance and insulin sensitivity. Diabetes Care. 2000;23:57-63.

\section{Figures}




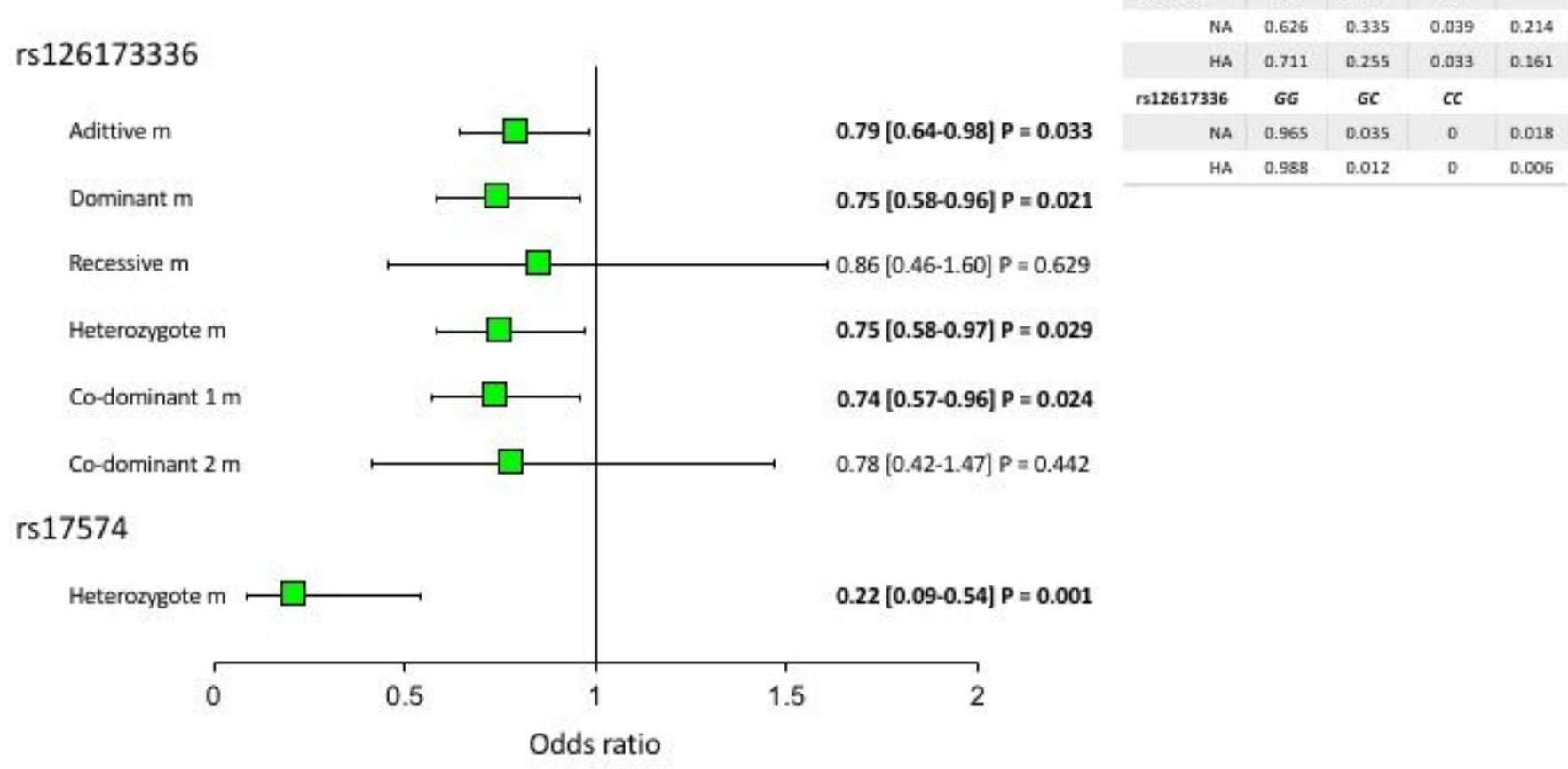

\section{Figure 1}

Association of DPP4 polymorphisms with hypoalphalipoproteinemia. MAF, minor allele frequency. All models were adjusted by age, sex, BMI, current smoking, physical activity, visceral abdominal fat, and triglycerides levels. The reference genotype was GG for rs 12617336 and AA for rs17574. Bold numbers indicate significant associations. 
A

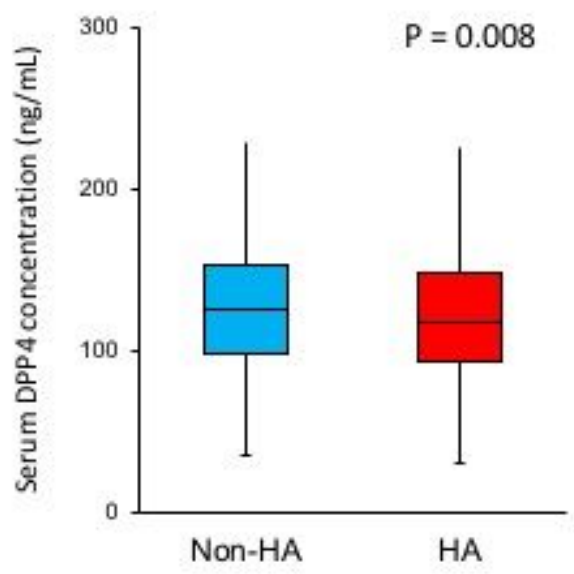

B

DPP4 rs17574 genotype

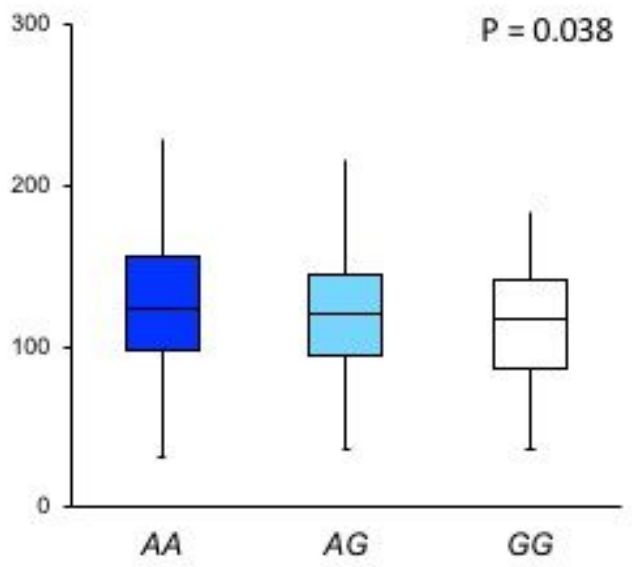

C

DPP4 rs17574 dominant model

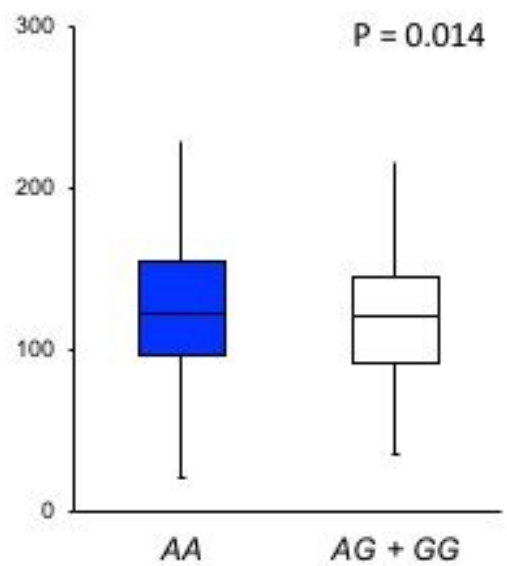

Figure 2

DPP4 concentration in the whole sample stratified by A) in non-hypoalphalipoproteinemia and hypoalphalipoproteinemia groups; $B$ ) in rs17574 genotypes; $C$ ) in non-carries (AA) and carriers (AG + GG) of rs17574 minor allele.

A

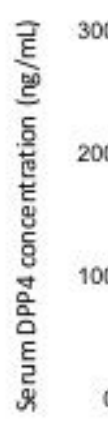

C

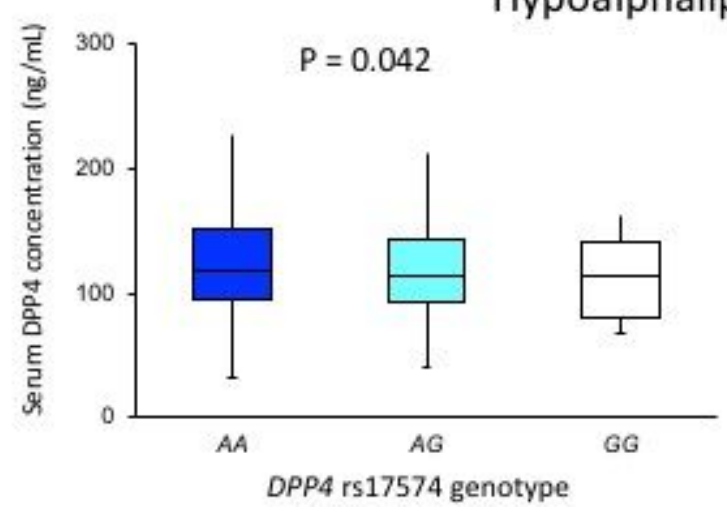

Non-hypoalphalipoproteinemia group

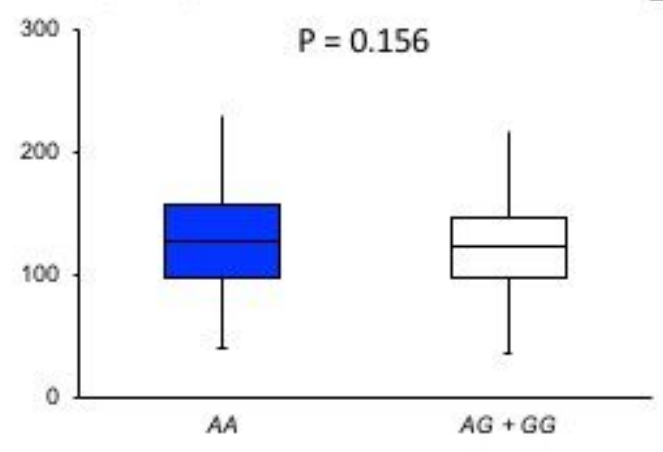

Hypoalphalipoproteinemia group

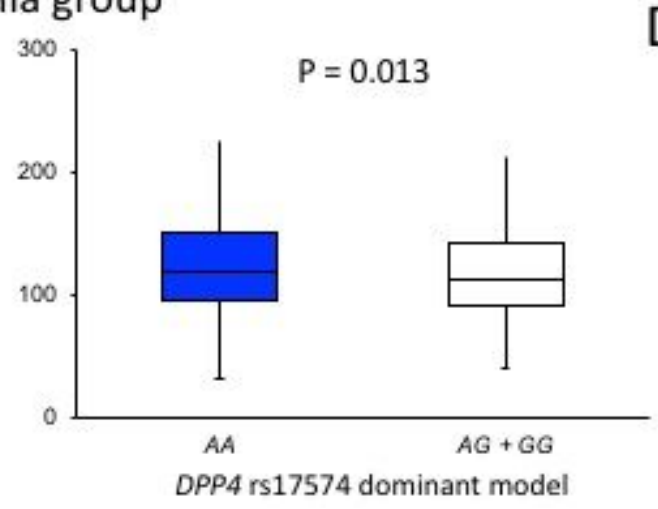




\section{Figure 3}

DPP4 concentration in non-hypoalphalipoproteinemia ( $A$ and $B$ ) and hypoalphalipoproteinemia ( $C$ and $D)$ groups, stratified by rs 17574 genotypes (A and $C$ ) and in non-carries (AA) and carriers (AG + GG) of rs17574 minor allele (B and D).

\section{Supplementary Files}

This is a list of supplementary files associated with this preprint. Click to download.

- CoverletterDPP4JTM.doc 